\title{
Statistical Analysis and Research on Common Defects of Precast Slab Girder Bridge
}

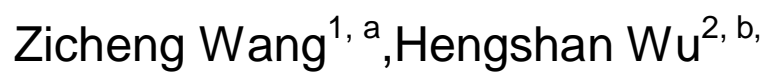

${ }^{1,2}$ School of Urban Construction,University of south China,Hengyang, Hunan 421001, PRa1165979548@qq.com, b2638813275@qq.com

${ }^{*}$ Corresponding author

Keywords: small and medium span; precast hollow slab; common defects; maintenance and reinforcement

Abstract: In this paper, the small and medium span precast hollow slab girder bridge in Dongguan city is taken as an example in order to statistically classify common defects of precast slab girder bridge respectively from the aspects of the bridge decking, upper structure and lower structure. Moreover, possible causes and disposal measures are analyzed from the perspectives of operation and maintenance. Basic technical data are provided for the research on precast slab girder bridge in terms of regular inspection, maintenance and reinforcement, and formulating conservation measures etc.

Dongguan, also known as "Guancheng", is located in the east coast of the Pearl River Estuary, south of Guangdong Province, China. It is the head of the "Four Tigers of Guangdong" and is referred to as "the Industrial Workshop of the World". With strong manufacturing and complete industrial system, Dongguan, whose manufacturing output accounts for more than $90 \%$ of the above-scale industrial output value, is one of the world's largest manufacturing bases. Dongguan features a mainly export-oriented economy. Especially in industry, most of the capital, raw materials and product sales are not separated from the international market, which means the road traffic in Dongguan city plays a vital role in urban development. Meanwhile, the healthy operation of bridge (especially the small and medium span bridge) is a guarantee of smooth traffic. Thus, it is strategically significant and has a far-reaching impact on Dongguan's advancement to research and analyze typical defects of small and medium span bridge, to grasp the degree of typical defects' damage to the bridge structure, and to master the technology and security of the bridge structure. 
Among the highways and urban roads and bridges in Dongguan, the concrete hollow slab bridge is the main structure type of the medium and small span bridge. Using precast hollow concrete slab bridge as an example, this paper statistically classifies typical defects of precast slab girder bridge respectively from the aspects of the bridge decking, upper structure and lower structure, at the same time, analyzes the causes and disposal measures.

\section{Typical defects, cause analysis and disposal measures of bridge deck system}

Bridge deck pavement defect

Vertical and transverse linear cracks emerge on the lane, or net cracks show up on the deck

Analysis of the reasons: when the temperature decreases, the shrinkage of asphalt concrete and the deformation of the upper part of the structure are easy to produce transverse cracks; the bridge deck is prone to map cracking because of the aging of asphalt, the poor grading, non-sufficient surfacing layer thickness and the infiltration of water.

Disposal measures: small cracks can be partially repaired; when cracks are too large, it is essential to check the structure below the pavement layer in case of injuring the bridge deck. In severe cases, the paving layer should be chiseled and relayed, the bridge deck waterproof layer should be repaired in time when damaged.

Asphalt concrete bridge deck pavement bleed and sag; wavy bumps and pot holes appear on the bridge deck pavement layer

Cause analysis: when the same position suffers from heavy traffic volume and too many vehicles for a long time, excessive amount of asphalt, plastic deformation, poor mixture gradation make it easy for the asphalt concrete pavement layer to bleed and sag; braking, starting, and overload, uneven asphalt spreading, and excessive amount of asphalt easily generate wavy bumps on the bridge deck pavement layer; the main reason is lack of repair and maintenance on deck cracking and net crack.

Disposal measures: as to the position of the bridge deck pavement bleeding, according to circumstances, coarse grain size aggregate can be sprinkled or be repaved; for larger holes, in order to prevent the damage of bridge deck, the bridge deck should be examined. Disposal methods for potholes are to dig potholes into the vertical rectangular groove wall, and to carry on layered filling and compaction with 
the same material as the original structure surrounded by brushing hot asphalt.

Bridge expansion joint defect

The expansion joint is blocked and impaired.

Cause analysis: maintenance is not implemented timely; the impurities in expansion joints haven't been cleaned timely and carefully; garbage or sediment accumulation piling up here result in expansion joint blockage.

Disposal measures: clean up the sundry and sediment in the expansion joints without delay; seriously damaged expansion joints are suggested be replaced by reasonable selection.

Pavement slab defects

There are pits, cracks and peelings on the pavement.

Analysis of reasons: defects of the pavement are mainly caused by the external forces such as the impact of motor vehicles and rolling. In addition, defects which just start to appear hasn't caused the attention of maintenance personnel, and repeated excavation is likely to be induced due to municipal pipeline construction. Each of them may also lead to pit slots, cracks, flake and other defects.

Disposal measures: perennial maintenance system on the pavement should be established; pavement damages should be repaired promptly with quality assurance. What's more, new materials which are energy-saving, environmental pressure-proof can also prevent the pavement damage during the pavement design and construction.

Defects of rails and fences.

Concrete of reinforced concrete fence columns suffer from aging, flaking, exposed tendons and deletion or steel fence columns are corroded and damaged.

Cause analysis: barrier defects mainly result from deficiency of necessary maintenance, vehicle impact, vandalism or disrepair. Large patches of defect on railings, in most cases, are usually caused by inappropriate employment of coating layer material or process. Small defect of the local area is usually induced by improper local handling with the coating surface .

Disposal measures: in terms of serious cracking or concrete's peeling of reinforced concrete railings, damaged parts of should be chiseled and completely fixed ; with regard to steel guardrail columns with mild corrosion, the rust should be removed. Then anti-rust paint should be brushed; corroded fence with seriously 
weakened section should be replaced. In addition, it is essential to establish a regular maintenance system with which the fence can enjoy regular maintenance.

\section{Defects of drainage system}

The rainwater collect pit is corroded and plugged; the drain hole is clogged; the bridge deck is covered with water; the drainage pipe is subject to corrosion, cracking or fracture.

Reason analysis: effective maintenance measures are deficient; debris or sediment plugs the drainage hole or set of wells that lead to impeded drainage and bridge deck dropsy; plastic aging, cast iron corrosion or vehicle impact damage cause water drain pipe to crack and fracture.

Disposal measures: the drainage pipe and the catch basin of the deck, if there is a blockage, should be timely cleared and often kept clear; measures should be taken in time to maintain, repair or replace damaged drainage pipe.

Lighting equipment, traffic marking defects

Posts are damaged; traffic signs and markings are damaged or missed.

Cause analysis: corrosion, the collision of vehicle, hurricane or man-made factors lead to the collapse of traffic signs and lamp posts; in addition, lack of conservation causes blurred and deleted markings.

Disposal measures: If corrosion reasons lead to the damage of a traffic sign or lamppost, protective treatment should be done for the benchmark. If it is because of hurricane or vehicle impact, the benchmark should be reinforced. Traffic line markings should often remain intact, clear and be re-coated regularly.

\section{Typical defects, cause analysis and disposal measures of upper structure}

The structure of the upper main bearing external loads of the defect.

The longitudinal and vertical cracks of the bottom surface of the precast hollow slab.

Cause analysis: small hinged joint defects, bearing defect, especially the bearing cavity lead to changes in the stress mode of bridge; pavement damage cause the bridge transverse stiffness, resulting in the beam and slab stress; free expansion joints plugging on beam and overload phenomena can cause precast hollow floor transverse to longitudinal cracks. 
Disposal measures: when the cracks are less and no more than the standard limit, we can only do the crack closure or grouting treatment; when the cracks are more and seam width exceeds the standard limit, we must do closed or grouting according to the actual situation of the cracks, and then take the measure of the steel plate or transverse pre-stressing; if the hollow plate defect is particularly serious, we can change the hollow plate. In addition, deck system, bearings, expansion joints at the relationship between defects must be timely treatment.

The surface of the precast hollow slab concrete suffers from honeycomb, surface voids, breakage, spalling and exposed reinforcement.

Cause analysis: the thickness of the concrete protection layer, the quality defect or suffering from the atmospheric corrosion of concrete are the reasons for the debonding of concrete material from the surface of the steel bar and the exposed tendons.

Disposal measures: we want to clean up the loose parts of hollow concrete slab honeycomb, surface voids, peeling and other defects, then use high strength grade concrete, cement mortar or other materials to repair.

Defects of bridge superstructure

Precast concrete hollow slab joints suffer from fracture, failure and seepage

Cause analysis: overloading phenomena, especially concentrated loading's seriously exceeding standard design, lead to inter plate hinge joints' cutting off; lacking of measures on defects such as pointing seepage and void exacerbates the damage of hinge joints; damage of bridge deck waterproofing layer, the water to seep into the hinge joint erosion, destruction of the hinge joints use function..

Disposal measures: we must strictly control the overloading of vehicles on the road, in the early stage of the defect to the joints of the joints and grouting treatment, strengthen preventive maintenance; we want to repair the bridge deck pavement waterproof layer, improve the drainage system, to prevent water penetration into the hinge joints.

\section{Defects of the support}

The support is cut, corroded and deformed.

Cause analysis: the vertical force of the bearing is small and the horizontal displacement is large, and the horizontal displacement of the bolt shear failure is caused by the horizontal friction force; In addition, the expansion joint damage 
causes the bridge head seepage and bearing corrosion which make the bearing loss its deformation function.

Disposal measures: for corroded bearing, rust-removing and rust-preventing measures should be taken and regular testing and maintenance is also needed; severely deformed bearing should be replaced.

\section{Typical defects, cause analysis and disposal measures of the substructure of the bridge}

Bridge pier defects

More overrun cracks with exposed tendons owing to concrete damage appear on the beam negative moment.

Cause analysis: Overloaded cars make support reaction force too large which results in the increase of pier top bending moment that leads to cracks; water infringement on the pier cap beam causes corrosion of the steel bar, concrete breakage and falling off.

Disposal measures: overloaded vehicles should be strictly prohibited; damaged joints should be repaired timely, timely closure of bridge pier cap beam cracks should be timely closed; corroded rebar and damaged concrete should be repaired and reinforced; preventive maintenance should be strengthened.

Abutment defect

More and larger vertical cracks appear on the $\mathrm{U}$ type abutment front wall and side walls

Cause analysis: uneven settlement of abutment foundation result in vertical cracks. In addition, with bridge pavement damage, water seep into the pavement back which lead to increasing soil pressure cause the defect of abutment.

Disposal measures: bridge pavement defects should be repaired timely, and the deck drainage system should be improved to prevent water from entering the abutment.

Pier foundation defect

The pier foundation suffer from serious erosion; the foundation partially hollow out

Cause analysis: the foundation is washed by the river and The pier near the 
construction leads to the bottom gap.

Disposal measures: if the foundation is scoured too deeply or local basement is emptied, stones, stone tablets and galvanized wire gab-ion should be filled immediately for maintenance.

Defect of cone slope protection

The cone slope masonry cracks and breaks, mortar of jointing partially falls off

Cause analysis: cone slope protection masonry cracking, and pointing mortar shedding are mainly due to the lack of necessary maintenance measures and foundation uneven settlement

Disposal measures: cone slope protection plant should be removed in time, and the shedding of jointing should be re-jointed; use mortar to mend slope cracking.

Bridge side wall defects

Cantilever wing wall leaks water and cracks

Cause analysis: the expansion joint damage causes rainwater infiltration; the abutment wall cracks under the pressure of soil.

Disposal measures: for concrete crack whose width is less than $0.15 \mathrm{~mm}$, use epoxy resin mortar to close it; for the crack whose width is greater than $0.15 \mathrm{~mm}$, use the low-pressure injection epoxy resin adhesive to repair.

\section{Conclusions}

Small and medium span bridges after long-term operation will inevitably produce a variety of defects, these defects on the durability of bridges and even safety have vary degrees of adverse effects. This article takes "The highway bridge technical condition assessment standard" ( JTG/T H21-2001) as the main line, based on the detection of a large number of precast concrete hollow slab bridge in Dongguan, the typical defect of the Dongguan city in the service precast slab bridge is described, and the causes of the defect are analyzed and the corresponding measures are given. In the lower part of the structure, the riverbed and the structure of the regulation are not too common, and there is no description. It provides basic technical data for the research on the regular inspection, maintenance and maintenance measures of the precast concrete hollow slab bridge in Dongguan, and 
provides reference for the research on the defect of the precast concrete hollow slab in other regions.

\section{References}

[1] People's Republic of China industry standards, Technical standard for inspection of urban bridge,DBJ/T 15-87-2011

[2]Highway Bridge Replacement and Rehabilitation Program.Federal Highway Administration.2011

[3]Zhejiang Provincial Department of transportation.Manual for Prevention and Repairing of Typical Defects in Medium and Small Span Bridges(2011)

[4] People's Republic of China industry standards,Code for Maintenance for Highwag Bridges and Culvers(TTG H11-2004)

[5] People's Republic of China recommended industry,Specification for Inspection and Evaluation of Load-bearing Capacity of Highway Bridges(JTG/T J21-2011) 